\title{
Successful reconstruction of the posterior cruciate ligament: assessment of posterior cruciate ligament footprints using an objective coordinate system
}

\author{
Ines Vielgut ${ }^{1} \cdot$ Andreas Weiglein $^{2} \cdot$ Stefan M. Biber ${ }^{1} \cdot$ Manuel Dreu $^{2} \cdot$ Andreas Leithner $^{1} \cdot$ Goria Hohenberger $^{1}$. \\ Patrick Sadoghi ${ }^{1}$
}

Received: 2 March 2020 / Accepted: 11 June 2020 / Published online: 17 June 2020

(c) The Author(s) 2020

\begin{abstract}
Introduction Anatomic cruciate ligament reconstruction is known to be correlated with better clinical results. The aim of the study was to provide a simple method to enable anatomic results in the setting of PCL reconstruction. We, therefore, assessed the tibial and femoral insertion site of the posterior cruciate ligament (PCL) by the use of an objective coordinate system in an anatomical study. We also sought to show reproducibility of these measurements using intra- and inter-observer coefficients. Materials and methods We studied 64 knees, previously preserved according to Thiel's technique. After proper preparation of the articular surfaces of both the tibiae and femora, photographs were taken according to a standardized protocol. PCL footprints were measured by the use of a coordinate system twice by two examiners. We evaluated these measurements by use of the Cohen's kappa inter- and intra-observer coefficient for two observers.

Results Tibial and femoral measurements of PCL footprints were generated with highly comparable inter- $(k=0.970)$ and intra-observer $(k=0.992)$ coefficients and may, therefore, be considered as highly reproducible.

Conclusion Our findings confirmed the reproducibility of defining PCL footprints using a coordinate system and may contribute to planning intraoperative graft-placement to ensure optimal conditions in the upcoming techniques for PCL reconstruction.
\end{abstract}

Keywords Anatomical study $\cdot \mathrm{PCL} \cdot \mathrm{Reconstruction} \cdot$ Tunnel positioning

\section{Introduction}

Injuries to the posterior cruciate ligament are reported to comprise approximately $3 \%$ of all knee ligament injuries in the general population $[6,9,14,16,20,24,29]$. PCL injuries rarely exist in isolation, and typically occur concurrently with other knee injuries, including ACL, medial collateral ligament (MCL), or posterolateral corner (PLC) injuries [11].

Anatomic single-bundle reconstruction of the PCL, using arthroscopic and radiographic reference points have focused

Ines Vielgut

ines.vielgut@medunigraz.at

1 Department of Orthopaedics and Trauma, Medical University of Graz, Auenbruggerplatz 5, 8036 Graz, Austria

2 Institute of Anatomy, Medical University of Graz, Harrachgasse 21/1 HG, 8010 Graz, Austria on current literature and more and more replaces the historical "isometric" reconstruction, where it was assumed that the femoral insertion point of the PCL maintains a fixed distance from a single point on tibia (tibial insertion site of the PCL) during range of motion (ROM). Reconstruction techniques which were based on this theory of an "isometric" PCL, have been reported to result in initial joint overconstraint and increased laxity over time, as it is accepted that the length of the ligament varies, depending on the tension on the ligament during ROM [10, 11].

Studies have described PCL reconstructions where the femoral and tibial residuals of the PCL are preserved to anatomically reinsert the PCL replacement graft $[1,3,4,12,13$, $16,17]$. This technique is hypothesized to enhance healing and transplant survival due to increased vascular ingrowth $[21,28]$. The most commonly reported negative impacts after PCL reconstruction are residual posterior laxity, flexion loss, and osteoarthritis as a long-term complication [24, 30]. 
Therefore, understanding PCL anatomy including ligament footprints is mandatory to achieve optimal results in PCL reconstruction $[4,15,19]$.

The purpose of this study was to precisely assess tibial and femoral insertion of the PCL using an objective coordinate system in an anatomical study on donated bodies to science.

Equally, we also sought to demonstrate the reproducibility of these measurements using intra- and inter-observer coefficients.

\section{Methods}

Ethical approval was obtained from the Ethics Committee of the Medical University of Graz. All bodies were donated to science, provided to the Department of Anatomy of the Medical University of Graz, under approval of the Anatomical Donation Program of the University of Graz, and the Austrian law for donations.

We studied 64 knees which were previously preserved using Thiel's technique [26]. The joints were taken from 30 male and 34 female bodies, with a mean age of 75 years at death (range, 41-101 years). The bodies had a mean height of $167.7 \mathrm{~cm}$ (range, 150-182 cm) and a mean weight of $67.3 \mathrm{~kg}$ (range, $46-115 \mathrm{~kg}$ ).

After the PCL was identified, both the cruciate ligaments, as well as the medial and lateral collateral ligaments were transected. Subsequently, the center of the tibial and the femoral footprint was visually identified and marked with a pen.

In case of a macroscopic double-bundle PCL, we chose the midpoint of these two bundles for assessment. After this, we photographed the tibiae according to criteria from a previously described protocol [19], and used an analogue setting to describe anatomical footprints of the PCL (Fig. 1a).

To optimize presentation of the intercondylar notch and the femoral PCL footprint, we rotated the knee from a strict posterior-anterior femoral view, in a $45^{\circ}$ external rotated position. This rotation was verified using a conventional graphometer circle (Fig. 2a).

Photographs were taken by both observers, and standardized photographs were printed to assess tibial and femoral footprints using a coordinate system, which was manually drawn.

\section{The coordinate system}

Both the tibial footprint of the PCL and coordinate values were drawn and measured on a strict cranio-caudal view. The coordinate system was oriented on the following anatomical landmarks: the zero values of the $X$ - and $Y$-axes

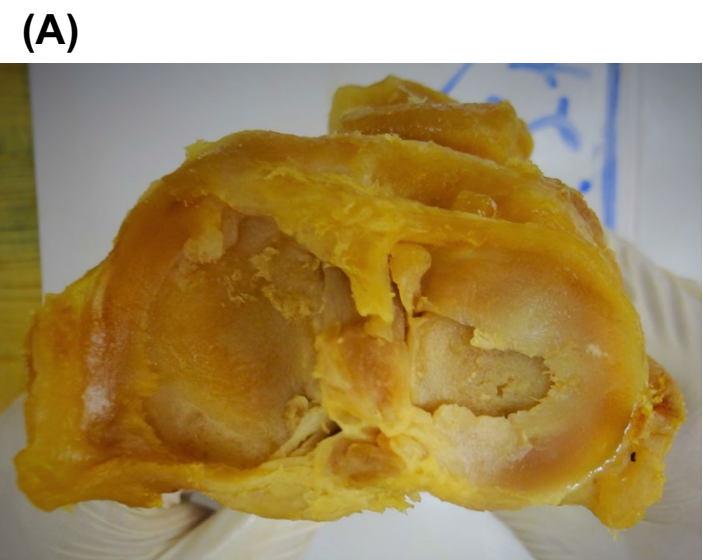

(B)

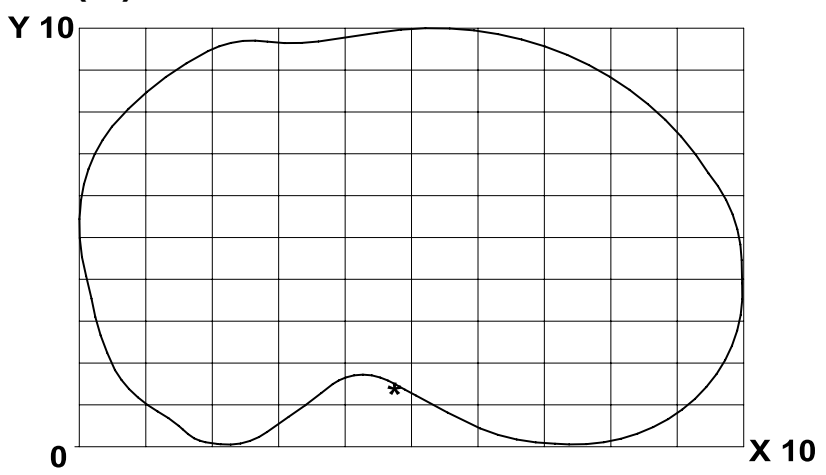

Fig. 1 a This picture shows the cranio-caudal view on the tibial plateau. All tibiae were photographed in this way to assess the tibial PCL insertion site by the use of an overlaid standardised coordinate system. b This sketch illustrates the standardised coordinate system overlaid onto a picture of a tibial plateau from a cranio-caudal view

represented the posteromedial corner of a rectangle drawn around the photograph of each tibial plateau. The length and width of the circumscribing rectangle was divided into ten equally large sections (Fig. 1b).

For the femur, the $X$-coordinate ranged from the cranial beginning of the condyle to its cranial end. The $Y$-coordinate ranged from the articulating femoral edge to the outer (lateral) edge of the lateral condyle (Fig. 2b).

Finally, the previously marked footprints of the PCL were defined using the sectioned coordinate system.

All preparations and measurements were performed twice by each observer, with an interval of 2 weeks between measurements to verify reproducibility of the technology.

First, we evaluated the mean values after four measurements, and second, we calculated Cohen's kappa coefficient for inter- and intra-observer reliability. The hereby presented methodology was previously published in an analogue setting by the study group for the ACL [19]. 
(A)

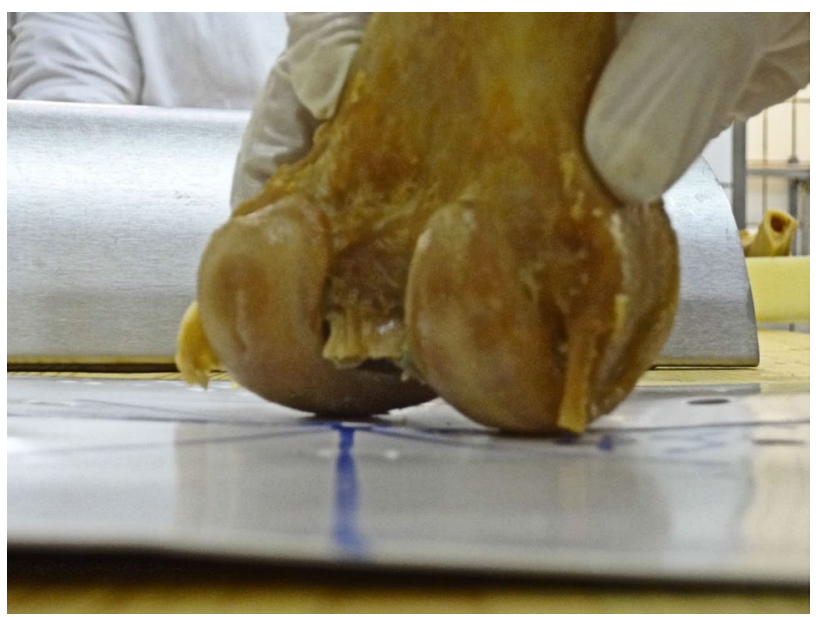

(B)

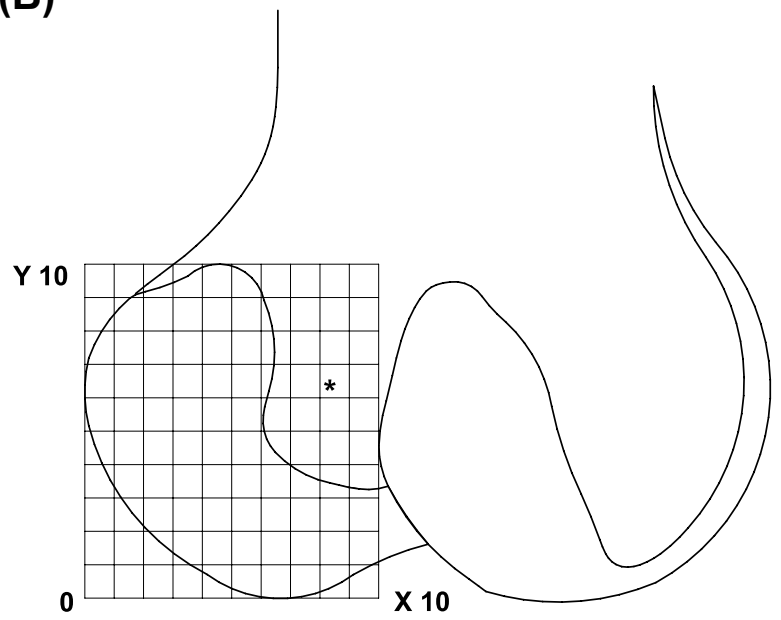

Fig. 2 a This picture illustrates the standardized photograph of a $45^{\circ}$ inwards rotated femur. $\mathbf{b}$ This sketch illustrates the standardised coordinate system which was overlaid onto a standardized photograph of a $45^{\circ}$ inwards rotated femur

\section{Statistical analysis}

The reliability of photographic production and the marking of insertion site positions on our coordinate system was evaluated using the Cohen's kappa coefficient for interand intra-observer reliability, for two observers. The kappa coefficient $(k)$ is a parameter of intra-observer agreement for continuous outcomes, and ranges from 1 (perfect agreement) to 0 (no agreement). An a priori power analysis and sample size estimation were performed according to a previous investigation of the study group [18]. For statistical analyses, SPSS version 16.0 for Windows was used, and a $P$-value $<0.05$ was considered statistically significant.
Table 1 Mean values of tibial and femoral insertion points of the PCL after four measurements by two observers

\begin{tabular}{lll}
\hline & Tibial footprint & Femoral footprint \\
\hline Observer 1 (first measurement) & $4.8 ; 1.4$ & $8.3 ; 6.6$ \\
Observer 2 (first measurement) & $4.7 ; 1.2$ & $8.4 ; 6.3$ \\
$\begin{array}{l}\text { Observer 1 (second measure- } \\
\text { ment) }\end{array}$ & $4.7 ; 1.3$ & $8.2 ; 6.2$ \\
$\begin{array}{l}\text { Observer 2 (second measure- } \\
\text { ment) }\end{array}$ & $4.7 ; 1.3$ & $8.3 ; 6.3$ \\
\begin{tabular}{l} 
Mean \\
\hline
\end{tabular} & $4.73 ; 1.3$ & $8.3 ; 6.35$ \\
\hline
\end{tabular}

\section{Results}

All tibial and femoral photographs were taken according to the described method, and were used to assess tibial and femoral attachment sites of the PCL using a coordinate system. The tibial and femoral coordinate points were measured with highly comparable inter-observer $(k=0.970)$ and intra-observer $(k=0.992)$ agreement, and may, therefore, be considered as highly reproducible measurements. Mean data after four measurements revealed a tibial insertion point $\mathrm{T}$ of $4.73 / 1.3$ and a femoral insertion point $\mathrm{F}$ of $8.3 / 6.35$, (Table 1).

\section{Discussion}

We observed that the tibial and femoral insertion site of the PCL was identified using our coordinate system, and we revealed a statistic significant intra- and inter-observer agreement (Cohen's kappa coefficient). Given the fact that the PCL is one of the major passive stabilizers of the knee joint, restraining both posterior translation and rotation of the tibia, it appears obvious that PCL injuries inevitably lead to knee joint instability and secondary osteoarthritis [7, 12, $19,23,24,28]$. Since the PCL is believed to play a more expansive role in providing rotational stability than previously thought, it is important to assess internal and external rotation stability, in addition to posterior tibial translation when considering PCL injury [9]. The particular consequences of these findings may generate higher detection rates and increased attention for new PCL injuries, given the fact that PCL injuries have been historically underdiagnosed and estimated due to the high percentage of primary asymptomatic patients [11].

Surgical PCL reconstruction may be performed using different techniques, including single- and double-bundle reconstruction [14, 21, 23, 27]. However, some authors have described the superiority of double-bundle PCL reconstruction when compared to single-bundle techniques. The loading patterns of the two bundles, respectively, PCL 
biomechanics in general, seem to have not been completely investigated up to now $[5,22,27,29]$.

Traditionally believed to function independently during flexion (anterolateral bundle) and extension (posteromedial bundle) of the knee, the recent literature has described relationships between the two bundles as more synergistic and co-dominant, based on simultaneous elongation during a forward lunge $[2,10,12,23,30]$. However, due to high variability and data heterogeneity in these studies regarding load patterns of the PCL during normal daily activities, a definitive role for the ligament and its functional bundles remains controversial [20,27].

Recent studies comparing anatomic single- versus double-bundle reconstruction techniques, suggest that the double-bundle technique closely approximates native knee kinematics, particularly beyond $90^{\circ}$ flexion, respectively, also immediately after implantation $[8,18]$. In contrast, Kim et al. determined no advantages of double-bundle over single-bundle PCL reconstruction, with respect to clinical outcomes or posterior knee stability [10]. Furthermore, singlebundle PCL reconstruction techniques have focused more on arthroscopic and radiographic reference points instead of the historical non-anatomic "isometric" reconstruction, with initial occurring joint over-constraint and progressive joint laxity described as complications [2, 3, 8, 10, 18, 20, 25, 27].

Our study has revealed that measurements of femoral and tibial insertion sites of the PCL are repeatable and reproducible, and therefore, accurate. Accordingly, this technique may represent a useful tool in preoperative planning for PCL reconstructions in the future. One possible application may be the preoperative determination of insertion sites using three dimensional-computed tomography reconstruction, with an overlaid coordinate system as presented here. The use of anatomical landmarks to create custom-made cutting blocks has already facilitated intraoperative handling in the setting of total knee arthroplasty [8].

This study has the following limitation: we assessed insertion sites of the PCL according to macroscopic findings, therefore, we did not distinguish between the insertion site of the anterolateral and the posteromedial bundle. However, we reiterate the following benefits: both PCL insertion sites were evaluated in a large number of human knees using standardized methods.

\section{Conclusions}

Our study demonstrates that measurements of the femoral and tibial insertion sites of the PCL are repeatable, reproducible and highly accurate.

Our precise measurements of the PCL's tibial and femoral footprint may facilitate intraoperative graft-placement in terms of determining the points of optimal repair for recreating the isometry of the PCL.

Acknowledgments Open access funding provided by Medical University of Graz.

Author contributions IV, SB and PS wrote the main manuscript text. MD supported the cadaver work, IV, MD, PS and SB prepared the figures. AW, AL and GH critically reviewed and revised the manuscript.

\section{Compliance with ethical standards}

Conflict of interest The authors declare no conflict of interest regarding the publication of this article.

Open Access This article is licensed under a Creative Commons Attribution 4.0 International License, which permits use, sharing, adaptation, distribution and reproduction in any medium or format, as long as you give appropriate credit to the original author(s) and the source, provide a link to the Creative Commons licence, and indicate if changes were made. The images or other third party material in this article are included in the article's Creative Commons licence, unless indicated otherwise in a credit line to the material. If material is not included in the article's Creative Commons licence and your intended use is not permitted by statutory regulation or exceeds the permitted use, you will need to obtain permission directly from the copyright holder. To view a copy of this licence, visit http://creativecommons.org/licenses/by/4.0/.

\section{References}

1. Ahn JH, Yang HS, Jeong WK, Koh KH (2006) Arthroscopic transtibial posterior cruciate ligament reconstruction with preservation of posterior cruciate ligament fibers: clinical results of minimum 2-year follow-up. Am J Sports Med 34(2):194-204

2. Anderson CJ, Ziegler CG, Wijdicks CA, Engebretsen L, LaPrade RF (2010) Arthroscopically pertinent anatomy of the anterolateral and postero- medial bundles of the posterior cruciate ligament. $\mathrm{J}$ Bone Jt Surg Am 94(21):1936-1945

3. Dargel J, Pohl P, Tzikaras P, Koebke J (2006) Morphometric sideto-side differences in human cruciate ligament insertions. Surg Radiol Anat 28(4):398-402

4. Del Buono A, Radmilovic J, Gargano G, Gatto S, Maffulli N (2006) Augmentation or reconstruction of PCL? A quantitative review. Knee Surg Sports Traumatol Arthrosc 21(5):1050-1063

5. Garbelotti Junior SA, Pelozo Junior O, Caldana RP, Ramalho A, Smith RL (2007) Experimental evaluation of 3-dimensional kinematic behavior of the cruciate ligaments. Clinics 2(5):619-626

6. Hirschmann MT, Müller W (1999) Complex function of the knee joint: the current understanding of the knee. Knee Surg Sports Traumatol Arthrosc 23:2780

7. Hosseini Nasab SH, List R, Oberhofer K, Fucentese SF, Snedeker JG, Taylor WR (2016) Loading patterns of the posterior cruciate ligament in the healthy knee: a systematic review. PLoS ONE. https://doi.org/10.1371/journal.pone.0167106

8. Johannsen AM, Anderson CJ, Wijdicks CA, Engebretsen L, LaPrade RF (2013) Radiographic landmarks for tunnel positioning in posterior cruciate ligament reconstructions. Am J Sports Med 41(1):35-42

9. Kennedy NI (2013) Kinematic analysis of the posterior cruciate ligament, part 1: the individual and collective function of 
the anterolateral and posteromedial bundles. Am J Sports Med 41:2828-2838

10. Kim SJ, Jung M, Moon HK, Kim SG, Chun YM (2011) Anterolateral transtibial posterior cruciate ligament reconstruction combined with anatomical reconstruction of posterolateral corner insufficiency: comparison of single-bundle versus double-bundle posterior cruciate ligament reconstruction over a 2- to 6-year follow-up. Am J Sports Med 39(3):481-489

11. Kim YK, Yoo JD, Kim SW, Park SH, Cho JH, Lim HM (2018) Intraoperative graft isometry in anatomic single-bundle anterior cruciate ligament reconstruction. Knee Surg Relat Res 30(2):115-120

12. LaPrade CM, Civitarese DM, Rasmussen MT, LaPrade RF (2015) Emerging updates on the posterior cruciate ligament: a review of the current literature. Am J Sports Med 43(12):3077-3092

13. Lee KH et al (2011) Combined posterolateral corner reconstruction with remnant tensioning and augmentation in chronic posterior cruciate ligament injuries: minimum 2-year follow-up. Arthroscopy 27(4):507-515

14. Milles JL et al (2017) Biomechanical comparison: single-bundle versus double-bundle posterior cruciate ligament reconstruction techniques. J Knee Surg 30(4):347-351

15. Naraghi A, White LM (2014) MR imaging of cruciate ligaments. Magn Reson Imaging Clin N Am 22(4):557-580

16. Nuelle CW et al (2017) Biomechanical comparison of five posterior cruciate ligament reconstruction techniques. J Knee Surg 30(4):347-351

17. Orakzai SH, Egan CM, Eustace S, Kenny P, O’Flanagan SJ, Keogh P (2010) Correlation of intra-articular osseous measurements with posterior cruciate ligament length on MRI scans. Br J Radiol 83(985):23-27

18. Race A, Amis AA (1998) PCL reconstruction: in vitro biomechanical comparison of "isometric" versus single and doublebundled "anatomic"' grafts. J Bone Jt Surg Br 80(1):173-179

19. Sadoghi P, Kropfl A, Jansson V, Müller PE, Pietschmann MF, Fischmeister MF (2011) Impact of tibial and femoral tunnel position on clinical results after anterior cruciate ligament reconstruction. Arthroscopy 27(3):355-364

20. Sadoghi P et al (2012) Evaluating the tibial and femoral insertion site of the anterior cruciate ligament using an objective coordinate system: a cadaver study. Injury 43:1771-1775
21. Schmeiser G, Hempfling H, Bühren V, Putz R (2001) The popliteal region: an anatomical study and a new approach to the tibial attachment of the posterior cruciate ligament. Surg Radiol Anat 23(1):9-14

22. Shelbourne KD, Davis TJ, Patel DV (1999) The natural history of acute, isolated, nonoperatively treated posterior cruciate ligament injuries. A prospective study. Am J Sports Med 27(3):276-283

23. Shin YS, Kim HJ, Lee DH (2017) No clinically important difference in knee scores or instability between transtibial and inlay techniques for PCL reconstruction: a systematic review. Clin Orthop Relat Res 475:1239

24. Spiridonov SI, Slinkard NJ, LaPrade RF (2010) Isolated and combined grade-III posterior cruciate ligament tears treated with double-bundle reconstruction with use of endoscopically placed femoral tunnels and grafts: operative technique and clinical outcomes. J Bone Jt Surg Am 93(19):1773-1780

25. Teng $\mathrm{Y}$ et al (2016) MRI analysis of tibial PCL attachment in a large population of adult patients: reference data for anatomic PCL reconstruction. BMC Musculoskelet Disord 17(1):384

26. Thiel W (1992) The preservation of the whole corpse with natural color. Ann Anat 174:185-195

27. Van Dommelen BA, Fowler PJ (1989) Anatomy of the posterior cruciate ligament. A review. Am J Sports Med 17(1):24-29

28. Wang CJ (2002) Injuries to the posterior cruciate ligament and posterolateral instabilities of the knee. Chang Gung Med J 25(5):288-297

29. Wang JH et al (2014) Effects of knee flexion angle and loading conditions on the end-to-end distance of the posterior cruciate ligament: a comparison of the roles of the anterolateral and posteromedial bundles. Am J Sports Med 42(12):2972-2978

30. Wijdicks CA et al (2013) Kinematic analysis of the posterior cruciate ligament, part 2: a comparison of anatomic single- versus double-bundle reconstruction. Am J Sports Med 41:2839-2848

Publisher's Note Springer Nature remains neutral with regard to jurisdictional claims in published maps and institutional affiliations. 